\title{
Minor erythema multiforme case report
}

\begin{abstract}
Erythema multiforme (EM) is an acute mucocutaneous disease that characterized by being self-limiting and immune-mediated; which is commonly associated with some medications and infections. It affects the mucosal surfaces and present clinically with varied forms ranging from minor localized mucosal lesions to a more severe, extensive mucocutaneous epithelial necrosis known as Stevens-Johnson syndrome (SJS) and toxic epidermal necrolysis (TEN). Here we report a case of EM in a 37year-old Sudanese female who developed the lesions after using a lip stick.
\end{abstract}

Keywords: erythema multiforme, lip stick, corticosteroids, hypersensitivity reaction
Volume 7 Issue 4 - 2017

\author{
Yousif I Eltohami, Nour E Alim, Amal H \\ Abuaffan \\ Faculty of Dentistry, University of Khartoum, Sudan
}

Correspondence: Amal H Abuaffan, Faculty of dentistry,

University of Khartoum, Sudan, Email amalabuaffan@yahoo.com

Received: May 17, 2017| Published: October 27, 2017

\section{Introduction}

Erythema multiforme (EM) is an acute self-limiting mucocutaneous disease that is considered to be a type IV hypersensitivity reaction associated with certain infections, medications, and other various triggers. ${ }^{1}$ About $50 \%$ of cases are idiopathic. Most notable causes are infectious agents and drugs. Infectious causes are more common in children and are implicated more commonly in EM. Herpes simplex infection is most common cause in young adults. ${ }^{2}$

Erythema multiforme is a disease of abrupt onset involving skin and mucous membranes where the lesions appear as red macules, papules and vesicles. Blistering and crusting may occur in the center of the lesion resulting in the characteristic target lesions. ${ }^{3}$ Nonspecific prodromal symptom such as fever malaise, myalgia, arthralgia, headache, sore throat, cough, nausea, vomiting may appear 1-14days before the skin lesions develop. ${ }^{4}$ The different types of EM include, minor form (mostly affecting one site, commonly oral mucosa with $<10 \%$ of body surface area are affected), major (cutaneous lesions and at least 2 mucosal sites, oral lesions are wide spread and severe $\&<10 \%$ of body surface area is involved), Stevens-Johnson syndrome (Main difference from major form is based on the typology and location of lesions and the presence of systemic symptoms $\&<10 \%$ of the body surface area is involved), Overlapping Stevens-Johnson syndrome and toxic epidermalnecrolysis (No typical targets, up to $10 \%-30 \%$ of the body surface area is affected) and Toxic epidermal necrolysis (characterized by epidermal detachment of $>30 \%$ of the body surface and widespread purpuric macules or flat atypical targets). ${ }^{5}$

Oral lesion appears along with skin lesion in $70 \%$ of the cases, they start as bullae on an erythematous base, but intact bullae are rarely seen by the clinician because they break rapidly. Intraoral lesions predominantly occur on the non-keratinized mucosae and are most common in the anterior part of the mouth, including the lips and tongue. ${ }^{6}$

We present a case of facial cosmetic induced EM minor and believe that the inducing chemical within the cosmetic is the triggering factor.

\section{Case scenario}

A 37years old Sudanese female came to the dental surgery clinic complaining from burning sensation, difficulty in eating and swallowing and multiple ulcerative lesions started 3weeks ago after use of lip sticks without history of previous attack.
Past medical history: patient generally fit without history of habit consumption.

Clinical examinations: Tender diffuse irregular shallow bloody ulcers affected the lower lip and bilateral buccal mucosa and oral commissures. Also bilaterally a racial pigementations were appeared on buccal mucosa (Figures 1-3). There were no detectable cutaneous target like lesions and no palpable submandibular or cervical lymphadenopathy.

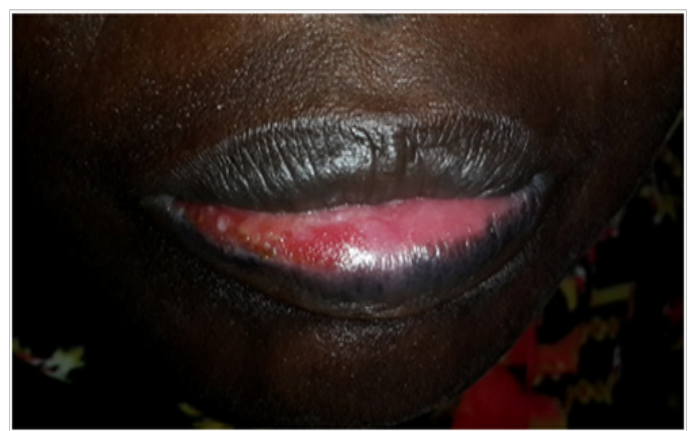

Figure I shows a diffuse irregular shallow bloody ulcers in the lower lip.

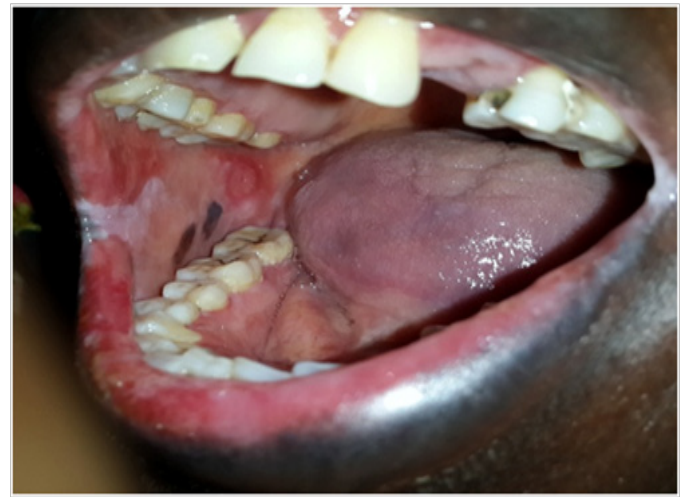

Figure 2 Shows diffuse irregular bloody ulceration in the right buccal mucosa.

Provisional diagnosis: Minor erythema multiforme.

Treatment: Systemic oral steroid at dose $1 \mathrm{mg} / \mathrm{kg}$ was prescribed for 2 weeks, after that dramatic improvement was observed clinically and gradual tapering started untill reached $5 \mathrm{mg}$ for 3 months as maintenance dose. 


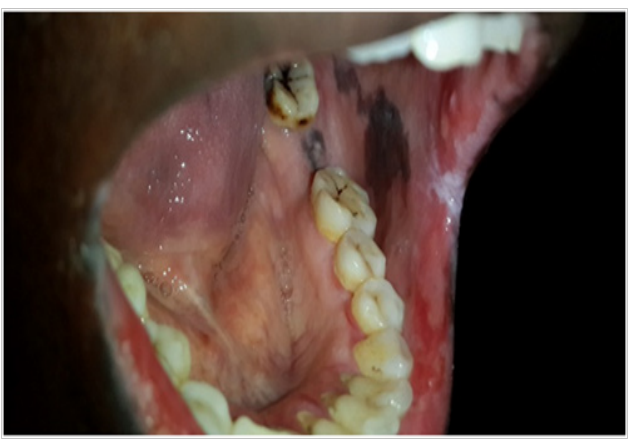

Figure 3 Shows diffuse irregular bloody ulcerations in the labial mucosa and in corner of the mouth.

\section{Discussion}

Erythema multiforme is a type of reactive mucocutaneous disorder, this reaction appears as a result of allergic host response to antigen exposure. ${ }^{7}$ In this case the allergic reaction triggered by the allergen that found in lipstick.

The presentation of EM ranges from self limited, mild form (EM minor) to progressive and aggressive form like EM major, Steven Johnson syndrome and Toxic epidermolysis necrosis, ${ }^{8}$ Kenneth in 1968 described an inflammatory oral disorder with oral lesions typical of EM. It has been suggested as a third category of EM by many investigators, known as oral EM that are characterized by typical lesions of EM but no target skin lesions. ${ }^{9,10}$

Oral lesions have different clinical presentations, ranging from diffuse oral erythema, to multifocal superficial ulcerations, thus the term multiforme. Initially, vesicles or bullae may be present, which rupture causing hemorrhagic crustations. Any area of the mouth may be involved, with buccal mucosa, palate, and tongue being most frequently affected. In most cases, lip lesions show hemorrhagic crustations. There may be mild to severe oral and perioral pain that may interfere with functional activities like speech, eating, swallowing and fluid intake, debilitating the health of the patient. Intraoral and perioral lesions heal without scarring, usually mild cases heal within 2-4weeks with local debridement, plenty of fluid intake and topical analgesics. Oral antihistamines and topical steroids provide symptomatic relief. Severe cases require intensive management with systemic steroids and intravenous fluid replacement, ${ }^{11}$ Recurrence is seen approximately in $20-25 \%$ of case with two to four episodes a year. ${ }^{12}$ In this case the clinical presentation goes with erythema multiforme minor, regarding the symptoms there was also burning sensation and difficulty in eating swallowing due to the oral and perioral ulcerations.

The diagnosis of EM is chiefly based on the history and clinical presentation, as histopathologic features and laboratory investigations are nonspecific. ${ }^{9,13}$

Corticosteroids are the most commonly used drugs in the management of EM, regardless of lack of evidence. ${ }^{9}$ This case treated by Systemic oral steroid at dose $1 \mathrm{mg} / \mathrm{kg}$ for 2 weeks, dramatic improvement was observed clinically, then gradual tapering was started. The patient followed up and no recurrence observed.

\section{Conclusion}

This case present erythema multiforme that triggered by allergen found in lipstick. Generally Systemic steroids are the main treatment though Supportive care and withdrawal of precipitating agents are equally important in managing erythema multiforme.

\section{Acknowledgements}

None.

\section{Conflict of interest}

The author declares no conflict of interest.

\section{References}

1. Sokumbi O, Wetter DA. Clinical features, diagnosis, and treatment of erythema multiforme:a review for the practicing dermatologist. Int $J$ Dermatol. 2012;51(8):889-902.

2. Marx RE, Stern D. Oral and Maxillofacial Pathology. A Rationale for Diagnosis and Treatment. Hanover Park, IL: Quintessence Pub; 2012.

3. Aburto C, Torres R, Caro A, et al. Sindrome de Stevens-Johnson asociado a infeccion por Mycoplasma pneumoniae y virus herpes. Folia Dermatol Peru. 2015;16(2):81-84.

4. Bashir T, Arti S, Jyoti G, et al. Erythema multiforme: a case report. Int $J$ Sci Stud. 2014;2(7):249-251.

5. Al-Johani KA, Fedele S, Porter SR. Erythema multiforme and related disorders. Oral Surg Oral Med Oral Pathol Oral Radiol Endod. 2007;103(5):642-654.

6. Farthing P, Bagan JV, Scully C. Mucosal disease series. Number IV. Erythema multiforme. Oral Dis. 2005;11:261-267.

7. P Sen, SH Chua. A case of recurrent erythema multiforme and its therapeutic complications. Ann Acad Med Singapore. 2004;33:793-796.

8. Scully C, Bagan J. Oral mucosal diseases: Erythema multiforme. British Journal of Oral and Maxillofacial Surgery. 2008;6(2):90-95.

9. Kohli PS, Kaur J. Erythema Multiforme-oral variant: case report and review of literature. Indian J Otolaryngol Head Neck Surg. 2011;63:9-12.

10. Joseph TI, Vargheese G, George D, et al. Drug induced oral erythema multiforme: A rare and less recognized variant of erythema multiforme. J Oral and Maxillofacial Pathology. 2012;16(1):145-148.

11. Williams PM, Cocklin RJ. Erythema multiforme. Review and contrast from Stevens Johnson syndrome/toxic epidermal necrolysis. Dent Clin North AM. 2005;49(1):67-76.

12. Kamala KA, Ashok L, Rajeshwari GA. Herpes associated erythema multiforme. Contemporary Clinical Dentistry. 2011;2(4):372-375.

13. Samim F, Auluck A, Zed C, et al. Erythema multiforme-A review of Epidemiology, Pathogenesis, Clinical Features, and Treatment. Dent Clin N Am. 2013;57(4):583-596. 